Pacific Journal of Mathematics

ON A CLASS OF TOPOLOGICAL ALGEBRAS

allan C. Cochran, R. Keown and C. R. Williams 


\section{ON A CLASS OF TOPOLOGICAL ALGEBRAS}

\section{A. C. Cochran, R. Keown and C. R. Williams}

This note introduces a class of topological algebras, called $A$-convex, which generalize the notion of locally $m$-convex algebras. They include a number of function space algebras which are not locally $m$-convex. Certain of these algebras admit a modified Gel'fand type representation in a space of vector-valued functions without invoking commutativity requirements. One seemingly obtains a new way of representing locally $m$-convex algebras. $A$-convex algebras are locally $m$ convex under the assumption of completeness of certain factor algebras in a suitable topology.

The definition of an $A$-convex algebra is given in $\S 2$ together with some basic results. We define a condition, $P$-complete, such that every $P$-complete, $A$-convex algebra is locally $m$-convex. A class of important functions algebras whose seminorms are defined by certain types of weight functions is defined in $\S 3$, see W. H. Summers [9]. Many of these are not locally $m$-convex, but are $A$-convex algebras. The definition and basic properties of an algebra of vector-valued functions where the index set is a completely regular Hausdorff space and the functions take values in (various) Banach algebras are given in $\S 4$. Finally, the result is obtained in $\S 5$ that each $A$-convex algebra is an inverse limit of $A$-normed (normed linear space with separately continuous multiplication) algebras. It is also shown that certain $A$-convex algebras can be represented as a subalgebra of an algebra of vector-valued functions. A sufficient condition for the representation to be valid is that $A$ be barrelled. It is shown by means of an example that barrelled is not necessary for this representation to be valid.

Some of our results are analogous to various others given by $\mathrm{P}$. D. Morris and D. E. Wulbert [7], G. R. Allan [1, 2], and R. M. Brooks $[4,5]$.

2. Basic definitions and results on multiplication. This paragraph is concerned with the introduction of some basic definitions and results on multiplications in a locally convex topological vector space. Let $A$ be a locally convex topological vector space over the complex numbers $K$ with a topology $T$ determined either by a family $N$ of absolutely convex neighborhoods of the origin or by a family $P$ of 
seminorms on the space. Assume that a multiplication is defined for $A$ with respect to which it is an algebra.

We wish to extend some of the additive concepts of locally convex topological vector spaces in the direction of multiplication.

Definition. 2.1 A subset $U$ of $A$ is said to be left (multiplicatively) absorbing if $a U$ is absorbed by $U$ for every $a$ in $A$. It is said to be right (multiplicatively) absorbing if $U a$ is absorbed by $U$ for every $a$ in $A$. It is said to be (multiplicatively) absorbing ( $m$ absorbing) if it is both left and right absorbing.

Let $p$ and $q$ denote elements of the family $P$ of seminorms of the algebra $A$.

Definition 2.2. The seminorm $p$ is said to $a b s o r b$ the seminorm $q$ if there exists a positive real number $M$ such that $q(x) \leqq M p(x)$ for every $x$ in $A$. The seminorms $p$ and $q$ are said to be conjugate if they are mutually absorbing. Let $[p]$ denote the class of all $q$ which are conjugate to $p$.

DeFINITION 2.3. The left-translate ${ }_{a} p$ (right-translate $p_{a}$ ) of any element $p$ of $P$ by the element $a$ of $A$ is the mapping from the algebra $A$ into the real numbers $R$ defined by ${ }_{a} p(x)=p(a x)\left[p_{a}(x)=p(x a)\right]$ for $x$ in $A$.

DEFINITION 2.4. A seminorm $p$ is left absorbing if it absorbs all of its left translates, right absorbing if it absorbs all of its right translates, and absorbing if it is both right and left absorbing.

It is clear that the class $[p]$ of $p$ is left-absorbing, right absorbing, and absorbing if and only if $p$ enjoys these respective properties.

We use the term locally convex algebra $A$ to designate a locally convex topological vector space $A$ with a multipication such that $A$ is an abstract algebra over the complex field $K$.

Definition 2.5. $A$ locally convex algebra $A$ is an $A$-convex algebra (absorbing convex algebra) if there exists a family $P$ of absorbing seminorms defining the topology of $A$.

Clearly every locally $m$-convex algebra is an $A$-convex algebra. The property of being $m$-absorbing is preserved with respect to taking convex hulls, inverse images under a homomorphism and images under a surjective homomorphism. The proof of the following theorem is 
straight-forward.

THEOREM 2.6. Any subalgebra of an A-convex algebra is A-convex. The product of A-convex algebras is again A-convex.

Let $A$ be an algebra without identity and let $A^{*}$ be the algebra obtained from $A$ by adjoining the identity. If $p$ is an absorbing seminorm on $A$ then the seminorm $p *$ defined by

$$
p *((x, \lambda))=p(x)+|\lambda|, x \in A, \lambda \in K,
$$

on $A^{*}$ is absorbing. Thus, every $A$-convex algebra can be topologically embedded in an $A$-convex algebra with identity.

Lemma 2.7. Let $p$ be a seminorm on the A-convex algebra $A$. Then there exist positive constants $M$ and $N$, depending on $x$, such that

$$
\begin{aligned}
& \text { (i) } \quad p(x y)={ }_{x} p(y) \leqq M p(y), \\
& \text { (ii) } p(y x)=p_{x}(y) \leqq N p(y),
\end{aligned}
$$

for any $y$ in $A$. The greatest lower bound of the $M$ for which (i) holds is denoted by ${ }_{p}\|x\|$ while the greatest lower bound of the $N$ for which (ii) holds is denoted by $\|x\|_{p}$.

The lemma follows directly from the definitions and implies that multiplication is continuous in the right (left) factor for a fixed left (right) factor.

The kernel $R(p)$ of a seminorm $p$ on the $A$-convex algebra $A$ is the set $\{x \in A: p(x)=0\}$. It follows immediately from Lemma (2.7) that $R(p)$ is a closed, two-sided ideal of $A$. Thus one can define the factor algebra $A \backslash R(p)$ on which $p$ induces a norm. Let $P$ be a family of seminorms defining the topology on an $A$-convex algebra $A$ such that $A \backslash R(\mathrm{p})$ is complete in the norm induced by $p$ for each $p \in R$. Then $A$ is said to be $P$-complete.

TheOREm 2.8. Let $A$ be an A-convex algebra which is P-complete in some family $P$ of absorbing seminorms defining the topology of $A$. Then there exists a representation $O(p)$ of $A$ by a Banach algebra $B$ for each seminorm $p$ of $A$.

Proof. Denote by $R$, rather than $R(p)$, the kernel of the seminorm $p$ of $A$. It follows from the definition that the factor algebra $A \backslash R$ is complete in the norm induced by $p$. The coset $x+R$ of $A \backslash R$ 
is denoted by $x^{\prime}$ for simplicity. The seminorm $p$ induces a norm $p^{p}$ on $A \backslash R$ which is defined for $x^{\prime}$ in $A \backslash R$ by

$$
p^{\prime}\left(x^{\prime}\right)=\inf \left\{p(y): \quad y \in x^{\prime}\right\} .
$$

Since $p$ is subadditive, $p^{\prime}\left(x^{\prime}\right)=p(y)$ for any $y \in x^{\prime}$. We note that

$$
\begin{aligned}
p^{\prime}\left(x^{\prime} y^{\prime}\right) & =p^{\prime}\left((x y)^{\prime}\right)=p(x y) \\
& \leqq{ }_{p}\|x\| p(y)={ }_{p}\|x\| p^{\prime}\left(y^{\prime}\right) .
\end{aligned}
$$

Thus multiplication is continuous in the right factor with respect to a fixed left factor in the norm $p^{\prime}$. Similarly it is continuous in the left factor with respect to a fixed right factor. It follows from a well-known theorem of Gel'fand that there exists a norm || on $A \backslash R$ equivalent to $p^{\prime}$ with respect to which $A \backslash R$ is a Banach algebra. The natural map $O(p)$ of $A$ into $A \backslash R$ is the required representation.

Corollary 2.9. Let $A$ be a P-complete A-convex algebra. Then $A$ is locally m-convex.

Examples 3. The first example gives a complete $A$-convex algebra which is not locally $m$-convex. Let $C_{b}(\mathbf{R})$ denote the algebra of bounded continuous complex-valued functions on the real numbers $\mathbf{R}$ (pointwise operations). Denote the set of strictly positive real-valued continuous functions on $\mathbf{R}$ which vanish at infinity by $C_{0}^{+}(\mathbf{R})$. The family of seminorms $\left\{p_{\phi}: \phi \in C_{0}^{+}(\mathbf{R})\right\}$ determine a locally convex linear topology $\beta$ on $C_{b}(\mathbf{R})$ where

$$
P_{\phi}(f)=\sup \{|f(x) \phi(x)|: x \in \mathbf{R}\}, f \in C_{b}(\mathbf{R}) .
$$

The space $\left(C_{b}(\mathbf{R}), \beta\right)$ is $A$-convex since

$$
P_{\phi}(f g) \leqq M(f) P_{\phi}(g), g \in C_{b}(\mathbf{R}),
$$

where $M(f)$ is the maximum of $|f|$. Completeness follows from Theorem 3.6 of [9]. It is easy to verify that each $p_{\phi}$ fails to be submultiplicative.

Suppose that $\left(C_{b}(\mathbf{R}), \beta\right)$ is locally $m$-convex and let $Q$ be a set of submultiplicative seminorms which define $\beta$. We may assume that $\max \left(q_{1}, \cdots, q_{n}\right) \in Q$ and $\lambda q_{1} \in Q$ whenever $q_{1}, \cdots q_{n} \in Q$ and $\lambda \geqq 1$. Thus, for $\phi \in C_{0}^{+}(\mathbf{R})$, there exists $q \in Q$ and $\psi \in C_{0}^{+}(\mathbf{R})$ such that

$$
(*) \quad V(\psi) \subseteq V(q) \subseteq V(\dot{\phi}),
$$

where $V(\psi)=\left\{f: p_{\psi}(f) \leqq 1\right\}$. Since $V(\psi) \subseteq V(\phi), \phi \leqq \psi$ (pointwise). Let $\theta \in \mathbf{R}$ with $0<\theta<\min (1, M(\psi))$. Then for some $x \in \mathbf{R}$ it follows that $\psi(x)=\theta \geqq \phi(x)$, and $\theta^{n}<\phi(x)$ where $n$ is a positive integer. 
Consider the function $f$ defined by

$$
f(y)=\left\{\begin{array}{c}
\frac{y-x+1}{\psi(y)}, x-1 \leqq y \leqq x \\
\frac{-y+x+1}{\psi(y)}, x<y \leqq x+1 \\
0, \text { otherwise. }
\end{array}\right.
$$

Then $f$ is well-defined since $\psi \in C_{0}^{+}(\mathbf{R})$ and $f \in C_{b}(\mathbf{R})$. But $p_{\psi}(f)=1$ and $p_{\phi}\left(f^{n}\right) \geqq f^{n}(x) \phi(x)=\theta^{-n} \dot{\phi}(x)>1$ which contradicts $(*)$ since $q$ is submultiplicative. Hence $\left(C_{b}(\mathbf{R}), \beta\right)$ is not locally $m$-convex.

This example is a special case of a weighted space (see W.H. Summers [9, 10]). By slight modifications of the arguments given here, other examples can be constructed using algebras of weighted functions. The representation of the last section is valid for this example.

A second example is obtained from the algebra $C[0,1]$ of all continuous complex valued functions on the closed interval $[0,1]$. A norm $p$ is defined on this algebra by

where $\quad \begin{aligned} p(f) & =\sup \{|f(x) \phi(x)|: x \in[0,1]\}, \\ & \phi(x)=\left\{\begin{array}{cl}x & 0 \leqq x \leqq 1 \mid 2 ; \\ 1-x & 1 \mid 2 \leqq x \leqq 1 .\end{array}\right.\end{aligned}$

Then $(C[0,1], p)$ is a normed linear space which is $A$-convex but not locally $m$-convex. This space is not complete and the topological completion is not an algebra.

Noncommutative examples may be obtained in the same manner as the first example where the range of the function space is a noncommutative space such as all bounded operators on a separable Hilbert space in the operator norm.

We now give a representation of an $A$-convex algebra in an algebra of vector-valued functions. The next section is concerned with the definition and basic properties of such an algebra.

4. Algebras of vector-valued functions. Suppose that $T$ is a completely regular Hausdorff space such that to each point $t$ of $T$ there corresponds a complex Banach algebra $B(t)$. Let $F$ denote the set of all vector-valued functions $f$ from $T$ to $\bigcup\{B(t): t \in T\}$ such that $f(t) \in B(t)$ for each $t \in T$ and such that the function $|f|$ defined by $\mid f /(t)=\|f(t)\|$ is continuous on $T(\|f(t)\|$ denoting the norm in $B(t))$. We consider any subset $H$ of $F$ which forms an algebra over $K$ with the usual pointwise definition of sum, product and scalar multiplication. Consider as a subbasis of neighborhoods of the origin in $H$ the 
sets $N(C, O)$, where $C$ is a compact subset of $T$ and $O$ an open subset of the real numbers $R$, defined by

$$
N(C, O)=\{f: f \in H, / f /(k) \in O \text { for all } k \in C\} .
$$

This system of neighborhoods determines a topology which by definition makes addition continuous and continuity of scalar multiplication is clear. This gives a topology on $H$ such that $H$ is a locally convex topological algebra. We consider $H$ with this topology in the remaining section.

The following two theorems and their corollaries generalize results which are well known if $T$ is compact and Hausdorff (and hence the elements of $F$ are bounded functions). They also extend a result of Morris and Wulbert [7] given in a similar setting where only commutative algebras are considered.

THeOREM 4.1. Let $H$ be a locally convex algebra as defined above. Suppose that

(i ) $H_{t}=\{f(t): f \in H\}=B(t)$ for all $t \in T$, and

(ii) The product $h f$ belongs to the closed ideal generated by $f$ for every choice of $f \in H$ and continuous real-valued function $h$ on $T$.

Then every closed (right, left, two-sided) ideal of $F$ is given by a set of the form $\left\{f \in H: f(t) \in I_{t}\right.$ for all $\left.t \in T\right\}$ where $I_{t}$ is a closed (right, left, two-sided) ideal in $B(t)$ for each $t \in T$. Conversely, every collection $\left\{I_{t}: t \in U\right\}$ (right, left, two-sided) ideals where $I_{t}$ is an ideal in $B(t)$ determines a closed (right, left, two-sided) ideal in $H$.

The proof is a direct generalization of one given by Naimark [8, $\S 26$, Subsection 2] of a similar theorem.

The following are now immediate:

COROLLARY 4.2. Every maximal closed (left, right, two-sided) ideal in $H$ consists of $\left\{f \in H: f(t) \in I_{t}\right\}$ where $I_{t}$ is a maximal closed ideal of $B(t)$.

CoRollary 4.3. If $B(t)$ is simple for each $t \in T$, then every closed two-sided ideal in $H$ consists of all $f \in H$ which vanish on some closed subset of $T$.

COROLLARY 4.4. If $B(t)$ is simple for each $t \in T$ then there is a one-to-one correspondence between the closed subsets of $U$ and closed ideals of $H$ via $G \rightarrow I_{G}=\{f \in H: f(G)=0\}$. 
COROLlary 4.5. If $B(t)$ is simple for each $t \in T$ there is a oneto-one correspondence between maximal closed ideals in $H$ and points of $T$.

Let $R(T)$ denote the algebra of all bounded real-valued functions which are continuous on $T$.

THEOREM 4.6. Let $T$ be a completely regular Hausdorff space such that

(i ) $H_{t}=B(t)$ for all $t \in T$;

(ii) For each $w \in B(t), w \in \overline{w B(t)}$;

(iii) $H$ is closed with respect to multiplication by elements in $R(T)$.

Then for any $g \in H$ and $a \in R(T)$, ag is an element of the closed ideal generated by $g$.

The proof is omitted.

5. Representations of A-convex algebras. An A-normed algebra is an $A$-convex algebra having a single absorbing norm defining the topology. The following theorem gives a generalization of a theorem of Michael [Proposition 2.7, 6].

THEOREM 5.1. A locally convex algebra is A-convex if and only if it is isomorphic to a subalgebra of a product of $A$-normed algebras.

The proof of this theorem is standard in view of the properties of $A$-convex algebras given in $\S 2$. The spaces $A \backslash R(p)$ used in Theorem (2.8) are used to make up the product space into which $A$ is embedded. Thus, an $A$-convex algebra is an inverse limit of $A$-normed algebras.

This theorem gives an alternate proof of Theorem (2.8). For if each factor algebra is complete in norm then each factor algebra is a Banach algebra and hence locally $m$-convex. Then $A$ is a subalgebra of a locally $m$-convex algebra. It follows that $A$ must be locally $m$-convex.

The second example of $\S 3$ shows that every $A$-normed algebra cannot be completed as an algebra. Note that if each factor algebra $A \backslash R(p)$ can be completed then $A$ is locally $m$-convex. This result follows from Theorem (5.1).

Suppose that $A$ is an $A$-convex algebra with $P$ a defining collection of seminorms. Let $\sigma_{p}$ denote the canonical quotient map of $A$ to $A \backslash R$ $(p)\left(=A_{p}\right)$ given in $\S 2$. If each $A_{p}$ has a completion we may 
consider $A_{p}$ as a subalgebra of its completion. For any finite subset $\left\{x_{1}, \cdots, x_{n}\right\}$ of $A$ and $\varepsilon>0$ the sets

$$
V_{p}\left\{x_{1}, \cdots, x_{n} ; \varepsilon\right\}=\left\{q \in P:\left|\left\|\sigma_{p}\left(x_{i}\right)\right\|-\left\|\sigma_{q}\left(x_{i}\right)\right\|\right|<\varepsilon, 1 \leqq i \leqq n\right\}
$$

give a base for a neighborhood system at $p \in P$. Then $P$ with this topology, $\mathscr{T}(P)$, is a completely regular Hausdorff space. In fact, the sets

$$
W\left(x_{1}, \cdots, x_{n} ; \varepsilon\right\}=\left\{(p, q) ;\left|\left\|\sigma_{p}\left(x_{i}\right)\right\|-\left\|\sigma_{q}\left(x_{i}\right)\right\|\right| \mid<\varepsilon, 1<i<n\right\}
$$

give a base of a uniformity inducing $\mathscr{T}(P)$. For each $p \in P$ we correspond the $A$-convex algebra $A_{p}$ and consider an algebra $H$ of vectorvalued functions from $P$ to the $A_{p}$ as described in $\S 4$. We show that if $A$ is barrelled then $A$ may be represented as a subalgebra of $H$. By proposition 4.3 of [6], a barrelled $A$-convex algebra is locally $m$ convex. However, the space $\left(C_{b}(\mathbf{R}), \beta\right)$, which is not barrelled, can be represented by our procedure. Also, this representation, which replaces a directed index set with a topological space, is seemingly different from the usual projective limit type representation for locally $m$-convex spaces [6].

Consider the Gel' fand map $G: A \rightarrow H$ where $G(x)(\mathrm{p})=\sigma_{p}(x)$. It is easy to verify that $\{G(x) \mid$ is continuous on $P$ for each $x$ in $A$. Since $P$ is Hausdorff, $G$ is one-to-one and it is clearly linear.

THEOREM 5.2. If $A$ is barrelled then $G$ is continuous.

Proof. It suffices to show that $G$ is continuous at 0 . A basic neighborhood of $G(0)=0$ is of the form $N\left(C, S_{\varepsilon}\right)$ where $C$ is compact in $P$ and $S_{\varepsilon}=\{r:|r|<\varepsilon\}$. The set, $M=\cap\left[p^{-1}[-\varepsilon / 2, \varepsilon / 2]: p \in C\right]$ is a barrel in $A$ [use the compactness of $C$ to see that $M$ is absorbing] and hence a neighborhood of 0 . But $G(M) \subseteq N\left(C, S_{\varepsilon}\right)$ so that $G$ is continuous.

THEOREM 5.3. The map, $G^{-1}$, is continuous.

Proof. Let $x \in A, G^{-1}(y)=x$. Then a basic neighborhood of $x$ is of the form

$V=x+\bigcap\left[p_{i}^{-1}\{(-\varepsilon, \varepsilon)\}: \varepsilon>0, p_{i} \in P, 1 \leqq i \leqq n\right] . \quad$ But the set $C$ $=\left\{p_{1}, \cdots, p_{n}\right\}$ is compact and $D=x+N\left(C, S_{\varepsilon}\right)$ is a neighborhood of $G(x)$ such that $G^{-1}(D)=V$. Hence $G^{-1}$ is continuous.

The last two theorems give the following modified Gel' fand type theorem. 
THEOREM 5.4. $A$ barrelled $A$-convex algebra $A$ can be represented as a subalgebra of an algebra of vector-valued functions as defined in $\S 4$.

For the space $\left(C_{b}(\mathbf{R}), \beta\right)$, the map $G$ of Theorem (5.2) in continuous. Since each $p$ is actually a norm, each quotient space is (setwise) $C_{b}(\mathbf{R})$. In the topology defined on $P$, if a set $M$ is compact then there exists $\phi \in C_{0}^{+}(\mathbf{R})$ such that $\psi \leqq \phi$ for each $p_{\psi} \in M$. Then for $V=p_{\phi}^{-1}(-\varepsilon, \varepsilon)$ it follows that $G(V) \subseteq N\left(M, S_{\varepsilon}\right)$ and the result follows. For function spaces of this nature, if each compact set is dominated by a single seminorm then $G$ is continuous and the representation is valid for the space.

If an $A$-convex algebra has an involution, *, one can define the concept of a subset being *-absorbing in a similar fashion to our previous definitions. The adjoint, $p^{*}$, of a seminorm $p$, is defined by $p^{*}$ $(x)=p\left(x^{*}\right)$ and an $A^{*}$-convex algebra can be defined to be an $A$-convex algebra which is defined by a family of absorbing seminorms, each of which absorbs its adjoint. Then Theorem (2.8) can be proven with the representation being in a symmetric algebra. Similarly, the representation of this section carries over to $A^{*}$-algebras with no difficulty, with the obvious restatements.

\section{REFERENCES}

1. G. R. Allan, On a class of locally convex algebras, Proc. London Math. Soc. (3) 17 (1967), 91-114.

2. A spectral theory for locally convex algebras, Proc. London Math. Soc. (3) 15 (1965), 399-421.

3. R. F. Arens, A generalization of normed rings, Pacific J. Math. 2 (1952), 455-471.

4. R. M. Brooks, On locally m-convex *-algebras, Pacific J. Math. 23 (1967), 5-23.

$5 . \quad$ On commutative locally m-convex algebras, Duke Math. J. 35 (1968), 257 -267 .

6. E. A. Michael, Locally multiplicatively-convex topological algebras, Mem. Amer. Math. Soc. 11 (1952).

7. P. D. Morris and D. E. Wulbert, Functional representations of topological algebras, Pacific J. Math. 22 (1967), 323-337.

8. M. A. Naimark, Normed rings, P. Noordhoff Ltd., Groningen, The Netherlands, 1964.

9. W. H. Summers, A representation theorem for biequicontinuous completed tensor products of weighted spaces, Trans. Amer. Math. Soc. 146 (1969), 121-131.

10. Weighted locally convex spaces of continuous functions, $\mathrm{Ph}$. D. dissertation, Louisiana State University, 1968.

Received May 21, 1969.

University of Arkansas Fayetteville, Arkansas

AND

Midwestern University Wichita Falls, Texas 



\section{PACIFIC JOURNAL OF MATHEMATICS}

EDITORS

H. SAMELSON

Stanford University

Stanford, California 94305

RichaRd PIERCE

University of Washington

Seattle, Washington 98105
J. DUGUNDJI

Department of Mathematics

University of Southern California

Los Angeles, California 90007

RICHARD ARENS

University of California

Los Angeles, California 90024

\section{ASSOCIATE EDITORS}

E. F. BECKENBACH

B. H. NeumanN

F. WOLF

K. YOSHIDA

\section{SUPPORTING INSTITUTIONS}

UNIVERSITY OF BRITISH COLUMBIA

CALIFORNIA INSTITUTE OF TECHNOLOGY

UNIVERSITY OF CALIFORNIA

MONTANA STATE UNIVERSITY

UNIVERSITY OF NEVADA

NEW MEXICO STATE UNIVERSITY

OREGON STATE UNIVERSITY

UNIVERSITY OF OREGON

OSAKA UNIVERSITY

UNIVERSITY OF SOUTHERN CALIFORNIA
STANFORD UNIVERSITY

UNIVERSITY OF TOKYO

UNIVERSITY OF UTAH

WASHINGTON STATE UNIVERSITY

UNIVERSITY OF WASHINGTON

AMERICAN MATHEMATICAL SOCIETY CHEVRON RESEARCH CORPORATION TRW SYSTEMS

NAVAL WEAPONS CENTER 


\section{Pacific Journal of Mathematics}

May, 1970

Johan Aarnes, Edward George Effros and Ole A. Nielsen, Locally compact

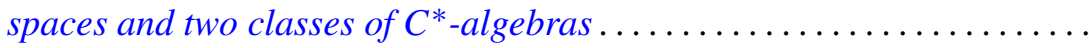

Allan C. Cochran, R. Keown and C. R. Williams, On a class of topological

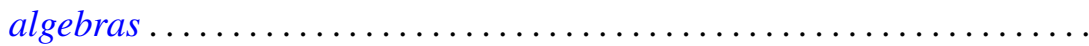

John Dauns, Integral domains that are not embeddable in division rings ....

Robert Jay Daverman, On the number of nonpiercing points in certain

crumpled cubes.....................................

Bryce L. Elkins, Characterization of separable ideals ................

Zbigniew Fiedorowicz, A comparison of two naturally arising uniformities

on a class of pseudo-PM spaces ...........................

Henry Charles Finlayson, Approximation of Wiener integrals of functionals

continuous in the uniform topology ........................

Theodore William Gamelin, Localization of the corona problem ...........

Alfred Gray and Paul Stephen Green, Sphere transitive structures and the

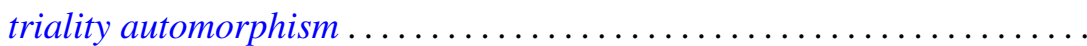

Charles Lemuel Hagopian, On generalized forms of aposyndesis ..........

J. Jakubík, On subgroups of a pseudo lattice ordered group ...............

Cornelius W. Onneweer, On uniform convergence for Walsh-Fourier

series..................................

Stanley Joel Osher, On certain Toeplitz operators in two variables ...

Washek (Vaclav) Frantisek Pfeffer and John Benson Wilbur, On the

measurability of Perron integrable functions............

Frank J. Polansky, On the conformal mapping of variable regions...

Kouei Sekigawa and Shûkichi Tanno, Sufficient conditions for a Riemannian manifold to be locally symmetric ...................

James Wilson Stepp, Locally compact Clifford semigroups ....

Ernest Lester Stitzinger, Frattini subalgebras of a class of solvable Lie

algebras ................................

George Szeto, The group character and split group algebras...

Mark Lawrence Teply, Homological dimension and splitting torsion

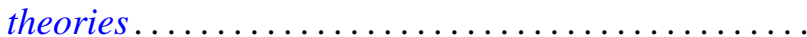

David Bertram Wales, Finite linear groups of degree seven. II ...

Robert Breckenridge Warfield, Jr., An isomorphic refinement theorem for

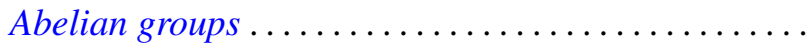

James Edward West, The ambient homeomorphy of an incomplete subspace

of infinite-dimensional Hilbert spaces................

Peter Wilker, Adjoint product and hom functors in general topology ...

Daniel Eliot Wulbert, A note on the characterization of conditional 